\title{
A 3-D four-wing attractor and its analysis
}

\author{
Zenghui Wang* \\ F'SATIE, Department of Electrical Engineering, Tshwane University of Technology, Pretoria 0001, South Africa and \\ Department of Automation, Shandong University of Science \& Technology, Qingdao 266510, China \\ Yanxia Sun, ${ }^{\dagger}$ Barend Jacobus van Wyk, and Guoyuan Qi \\ F'SATIE, Department of Electrical Engineering, Tshwane University of Technology, Pretoria 0001, South Africa \\ Michael Antonie van Wyk \\ School of Electrical and Information Engineering, University of the Witwatersrand, Johannesburg 2000, South Africa \\ (Received on 14 April, 2009)
}

\begin{abstract}
In this paper, several three dimensional (3-D) four-wing smooth quadratic autonomous chaotic systems are analyzed. It is shown that these systems have a number of similar features. A new 3-D continuous autonomous system is proposed based on these features. The new system can generate a four-wing chaotic attractor with less terms in the system equations. Several basic properties of the new system is analyzed by means of Lyapunov exponents, bifurcation diagrams and Poincare maps. Phase diagrams show that the equilibria are related to the existence of multiple wings.
\end{abstract}

Keywords: Chaos; four-wing attractor; Lyapunov exponents; bifurcation.

\section{INTRODUCTION}

Since Lorenz discovered a simple three-dimensional smooth autonomous chaotic system in 1963 [1], it was found that chaos is very useful in many application fields such as engineering, medicine, secure communications, and so on. Much research was done in this field [2, 3]. Creating a chaotic system with a more complicated topological structure such as a multi-scroll or multi-wing attractor, therefore, becomes a desirable task and sometimes a key issue for many engineering applications. In this endeavor, there are two major thrusts: generalizing Chua's circuits with multi-scroll attractors and generalizing the Lorenz system with multi-wing attractors. In efforts to generalize Chua's circuit [4] to produce multi-scroll attractors, several effective techniques have been developed, including generalized Chua's circuits and cellular neural networks [5, 6]. In $[4,5,7]$, the piecewise-linear (PWL) function method was utilized, which can increase the number of equilibria by adding breakpoints. A sine-function approach was then proposed for creating multi-scroll chaotic attractors [8]. Later, a stair function was used for generating 3D-grid-scroll attractors $[9,10]$. More recently, several different nonlinear functions including switching, hysteresis and saturated functions were utilized for creating chaotic attractors with multi-merged basins of attraction, or with multi-scroll attractors [11-14]. Note that the aforementioned methods for generating multi-scroll attractors have some common characteristics [15, 16]:

(i) The nonlinearities of these systems are usually not smooth functions; they are either piecewise-linear continuous functions or discontinuous ones such as the staircase function, switching function, and hysteresis-series function. (ii) The basic techniques either increase the number of equilibria via PWL functions with more breakpoints, or use

*Electronic address: wangzengh@gmail.com

${ }^{\dagger}$ Electronic address: sunyanxia@gmail.com stair or hysterisis functions to realize equilibrium jumping. (iii) The number of scrolls equals the number of equilibria. (iv) The basic shape of the attractors is cyclic, called a scroll.

Another major thrust has been the generalization of the Lorenz system [17]. Recently some new chaotic systems were proposed, including the Chen system, the generalized Lorenz system family, and the hyperbolic-type of generalized Lorenz canonical form [18-20]. Some four-dimensional chaotic systems were also presented, which have more complicated dynamic properties than three-dimensional chaotic systems, such as the system proposed in [21]. It can be seen that the characteristics of generalized Chua's circuits are different from the generalized Lorenz systems. For example, the nonlinearities of these systems are usually smooth functions, the number of wings is not equal to the number of equilibria and the basic shape of the attractors is a butterfly, called a wing [15, 22]. Qi proposed two four-wing chaotic attractors produced by 4-D systems with complicated structure $[16,22]$.

In fact, most of the multi-scroll attractors were generated by increasing the breakpoints in the non-linearity. Recently, a four-wing or three-wing butterfly attractor was generated from a three-dimensional system [23] by relying on two embedded state-controlled binary switches. However, these systems are usually not smooth systems.

It would be very exciting to construct a lower-dimensional chaotic system which has less terms in the system equations, but with a complex attractor structure. To generate multi-wing chaotic attractors from a three-dimensional (3D) smooth system remains a technical challenge, especially if a simple structure is a pre-requisite. In this paper, we analyze several proposed smooth quadratic autonomous 4wing chaos systems and present a new 4-wing chaotic system whose number of linear and quadratic terms is less than existing 4-wing chaotic systems. In the following sections, the new system is investigated by means of the Lyapunov exponent spectrum, Poincare maps and bifurcation diagrams. 


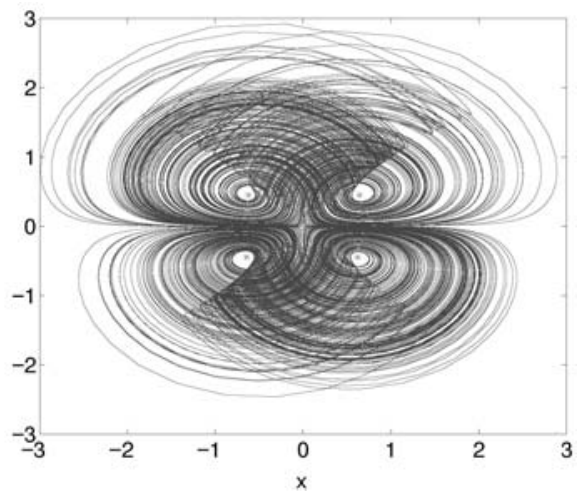

(a) Projection on the $x-y$ plane

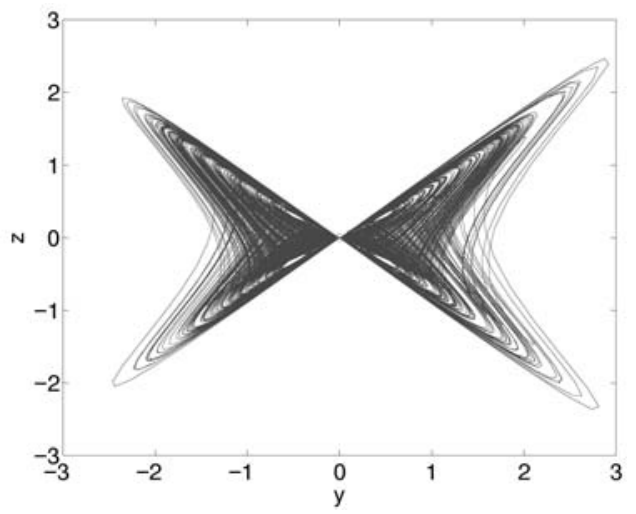

(c) Projection on the $y-z$ plane

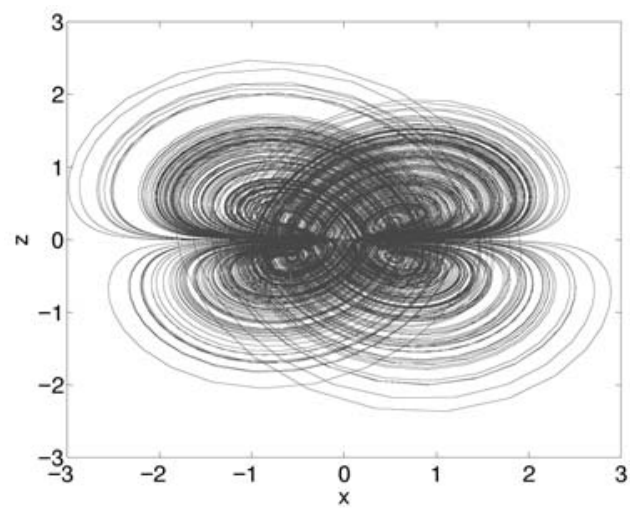

(b) Projection on the $x-z$ plane

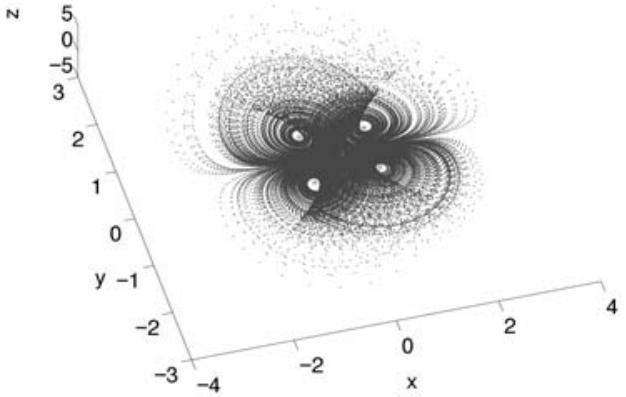

(d) 3-D view by '? in the $x-y-z$ space

FIG. 1: Four-wing chaotic attractor, with $a=0.2, b=-0.01, c=1, d=-0.4, e=-1.0$ and $f=-1$.

\section{3-D FOUR-WING SMOOTH AUTONOMOUS CHAOTIC} SYSTEMS

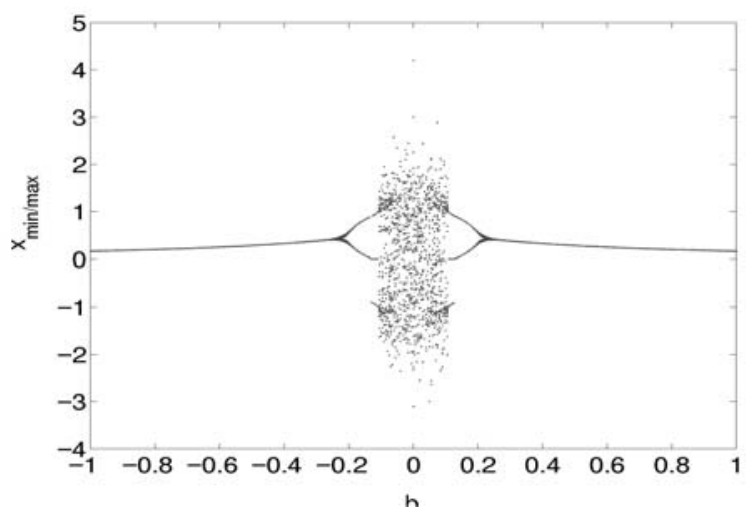

FIG. 2: The bifurcation diagram of the system (6) with respect to $b$, and with $a=0.2, c=1, d=-0.4, e=-1.0$ and $f=-1$.

In $[24,25]$, a three-dimensional smooth quadratic autonomous system which seemingly can produce a four-wing attractor was proposed. At first, it was believed that this system could produce a four-wing chaotic attractor, termed a four-scroll attractor but this was then later shown by the same authors to be a numerical artifact. It was not a real four-wing chaotic attractor but consisted of two coexisting and closely located double-wing attractors [26]. In [27], a $3-\mathrm{D}$ autonomous quadratic system was reported, which can generate a single four-scroll attractor. For the simplicity of comparison, the system is parameterized as

$$
\begin{aligned}
& \dot{x}_{1}=a_{1} x_{1}-y_{1} z_{1}+a_{2}, \\
& \dot{y}_{1}=b_{1} y_{1}+x_{1} z_{1}, \\
& \dot{z}_{1}=c_{1} z_{1}+x_{1} y_{1},
\end{aligned}
$$

where $a_{1}, a_{2}, b_{1}, c_{1}$ are real constants. If

$$
\left\{\begin{array}{l}
b_{1} c_{1}>0, \\
a_{1} \neq 0, \\
a_{1} \sqrt{b_{1} c_{1}}<\min \left(-a_{2}, a_{2}\right) \quad \text { if } b_{1}>0, \\
a_{1} \sqrt{b_{1} c_{1}}>\max \left(-a_{2}, a_{2}\right) \text { if } b_{1}<0,
\end{array}\right.
$$

there are five equilibria in this system, given by

$$
\begin{aligned}
S_{0} & =\left(-\frac{a_{2}}{a_{1}}, 0,0\right), \\
S_{1,2} & =\left(p, \pm \sqrt{\frac{\left(-a_{2}-a_{1} p\right) b_{1}}{p}} p, \pm \sqrt{\left.\frac{\left(-a_{2}-a_{1} p\right) b_{1}}{p}\right)}\right. \\
S_{3,4} & =\left(-p, \mp \sqrt{\frac{\left(a_{2}-a_{1} p\right) b_{1}}{p}} p, \pm \sqrt{\left.\frac{\left(a_{2}-a_{1} p\right) b_{1}}{p}\right)},\right.
\end{aligned}
$$

where $p=\sqrt{b_{1} c_{1}}$. As can be seen from the equilibria, there is no trivial equilibrium caused by the constant input of first 
equation in (1). Consider the following transformation of variables:

$$
x_{1}=x_{2}-\frac{a_{2}}{a_{1}} .
$$

The system (1) can be reformulated as

$$
\begin{aligned}
& \dot{x}_{2}=a_{1} x_{2}-y_{1} z_{1}, \\
& \dot{y}_{1}=b_{1} y_{1}-\frac{a_{2}}{a_{1}} z_{1}+x_{2} z_{1}, \\
& \dot{z}_{1}=c z_{1}-\frac{a_{2}}{a_{1}} y_{1}+x_{2} y_{1},
\end{aligned}
$$

which has a trivial equilibrium and is equivalent to system (1). Another example is given by

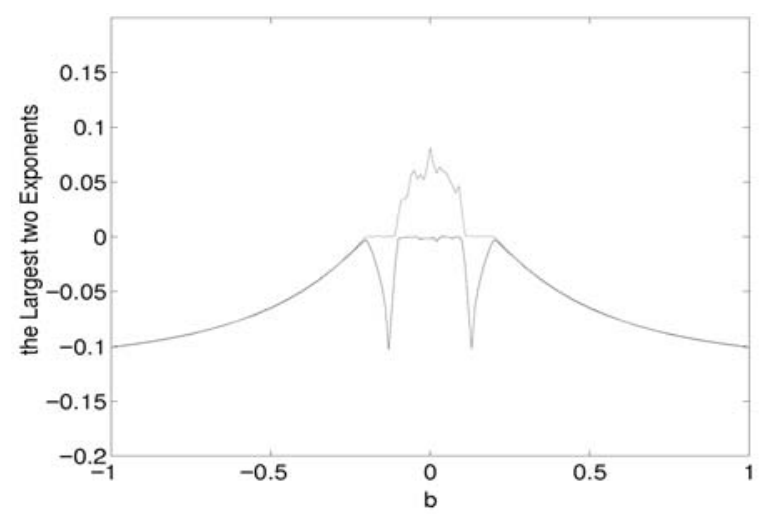

FIG. 3: The maximum Lyapunov exponent spectrum of the system (6) with respect to $b$, and with $a=0.2, c=1, d=-0.4, e=-1.0$ and $f=-1$.

$$
\begin{aligned}
& \dot{x}_{1}=a_{1} x_{1}+a_{2} y_{1}+y_{1} z_{1}, \\
& \dot{y}_{1}=b_{2} y_{1}-x_{1} z_{1}+b_{23} y_{1} z_{1}, \\
& \dot{z}_{1}=c_{3} z_{1}-x_{1} y_{1} .
\end{aligned}
$$

When $a_{1}=0.5, a_{2}=0.15 ; b_{2}=-12.2, b_{23}=1.0, c_{3}=$ -8.79 , the system has a real four-scroll attractor with eight cross product terms on the right [28].

Qi [15] introduced a 3-D quadratic autonomous system

$$
\begin{aligned}
& \dot{x}_{1}=a_{1}\left(y_{1}-x_{1}\right)+e_{1} y_{1} z_{1}, \\
& \dot{y}_{1}=c_{1} x_{1}+d_{1} y_{1}-x_{1} z_{1}, \\
& \dot{z}_{1}=-b_{1} z_{1}+x_{1} y_{1},
\end{aligned}
$$

called the Qi 3-D four-wing system, which can generate two coexisting single-wing chaotic attractors and a pair of diagonal double-wing chaotic attractors. The system can also generate a four-wing chaotic attractor with very complicated topological structures over a large range of parameters.

Recently, Chen [29] presented a new three-dimensional smooth quadratic autonomous chaotic system,

$$
\begin{aligned}
& \dot{x}_{1}=a_{1} x_{1}+k y_{1}-y_{1} z_{1}, \\
& \dot{y}_{1}=-b_{1} y_{1}-z_{1}+x_{1} z_{1}, \\
& \dot{z}_{1}=-x_{1}-c_{1} z_{1}+x_{1} y_{1} .
\end{aligned}
$$

which can evolve into periodic and chaotic orbits in case of different parameters. When proper parameters are chosen, a single four-wing attractor and a single three-wing attractor appears.

As can be seen from systems (2), (3), (4) and (5), there are a number of similar features common to these chaotic systems:

(1)There is at least one quadratic term in every equation, which means there is at least three quadratic terms in one system.

(2)There are five equilibria when these chaotic systems display four wings.

(3)There are at least four linear terms, and there is at least one linear term in every equation of these systems.

The logical question is whether there is any system exhibiting similar behavior, but with less linear and quadratic terms than these systems.

\section{A NEW 3-D FOUR-WING SMOOTH AUTONOMOUS CHAOTIC SYSTEMS}

Based on the above features, a new simpler chaotic system

$$
\begin{aligned}
& \dot{x}=a x+c y z, \\
& \dot{y}=b x+d y-x z, \\
& \dot{z}=e z+f x y,
\end{aligned}
$$

is proposed. Here $a, b, d, e \in R, c>0$ and $f<0$ are all constants, $c f \neq 0$, and $x, y, z$ are the state variables. If the system is dissipative, $\nabla V=\frac{\partial \dot{x}}{x}+\frac{\partial \dot{y}}{y}+\frac{\partial \dot{z}}{z}=a+d+e$ should be less than zero, that is, $a+d+e<0$. This implies that a volume element $V_{0}$ is contracted by the flow into a volume element $V_{0} e^{(a+d+e) t}$ in time $t$.

Theorem 1 If $b=0$, the system (6) can not generate a fourwing chaotic attractor.

Proof: This theorem can be proved according to different cases in parameter space.

Case 1: $c<0$

If $b=0$, consider the first and the second equations of (6), i.e.

$$
\begin{aligned}
& \dot{x}=a x+c y z, \\
& \dot{y}=d y-x y .
\end{aligned}
$$

By multiplying both sides of (7) by $x$ and of (8) by $c y$, respectively, the two equations become

$$
\begin{aligned}
\dot{x} x & =a x^{2}+c x y z, \\
c \dot{y} y & =c d y^{2}-c x y z .
\end{aligned}
$$

By adding both sides of (9) and (10), one obtains

$$
\dot{x} x+c \dot{y} y=a x^{2}+c d y^{2} .
$$

Equation (11) is equivalent to

$$
\frac{d\left(x^{2}+c y^{2}\right)}{d t}=2 d\left(x^{2}+c y^{2}\right)+(2 a-2 d) x^{2} .
$$


Solving the above equation yields

$$
x^{2}+c y^{2}=e^{2 d t}\left(\int_{0}^{t} e^{-2 d t}(2 a-2 d) x^{2} d \tau+\left(x_{0}^{2}+c y_{0}^{2}\right)\right),
$$

where $x_{0}$ and $y_{0}$ are the initial state of system (6).

If $a>d$ and $\left|x_{0}\right|>\sqrt{-c}\left|y_{0}\right|$, according to (13), one obtains

$$
x^{2}+c y^{2}>0 \Rightarrow|x|>\sqrt{-c}|y| .
$$

If $\left|x_{0}\right|>\sqrt{-c}\left|y_{0}\right|$ and $z_{0} \neq 0$, then for any time $t>0$, the system trajectory in the $x-y$ plane will never travel from one domain $x>0$ to another, $x<0$, or vice versa. That is, if $x_{0}>0$, then there will always be $x(t)>0$ for $t>0$; if $x_{0}<0$, then there will always be $x(t)<0$ for $t>0$. The same is for $a<d$ and $\left|x_{0}\right|<\sqrt{-c}\left|y_{0}\right|$.

If the system can generate a four-wing attractor, then the attractor will not depend on the initial state, that is , the initial state value can be chosen arbitrarily, such as $\left|x_{0}\right|>\sqrt{-c}\left|y_{0}\right|$ or $\left|x_{0}\right|<\sqrt{-c}\left|y_{0}\right|$. It conflicts with the above analysis, so it is impossible to be a four-wing attractor when $c<0$ and $b=0$.

Case 2: $c>0$

If $f>0$, the same result can be got according to the first and third equations of system (6).

If $f<0$, the same result can also be got according to the second and third equations of system (6).

The proof is thus completed.

\subsection{Equlibria}

The equilibria of system (6) can be easily derived by solving the three equations $\dot{x}=0, \dot{y}=0$, and $\dot{z}=0$. There is one trivial equilibrium.

Let

$$
\begin{aligned}
y_{e} & =\sqrt{\frac{a e}{c f}}, z_{e}^{1}=\frac{b c+\operatorname{sign}(a) \sqrt{b^{2} c^{2}-4 a c d}}{2 c}, z_{e}^{2} \\
& =\frac{b c-\operatorname{sign}(a) \sqrt{b^{2} c^{2}-4 a c d}}{2 c},
\end{aligned}
$$

where $\operatorname{sign}()$ is a sign function. If $\frac{a e}{c f}>0, b^{2} c^{2}-4 a c d>0$ and $c \neq 0$, there are four nontrivial equilibria:

$$
S_{1,2}=\left(\mp \frac{c}{a} y_{e} z_{e}^{1}, \pm y_{e}, z_{e}^{1}\right), S_{3,4}=\left(\mp \frac{c}{a} y_{e} z_{e}^{2}, \pm y_{e}, z_{e}^{2}\right)
$$

In this case, system (6) has five equilibria (including the zero equilibrium). It means system (6) is not topologically equivalent to the generalized Lorenz canonical form (GLCF) [20] which have three-equilibria at most.

Remark 1 If $\frac{a e}{c f}<0, b^{2} c^{2}-4 a c d<0$, there is no nontrivial equilibrium for system (6).

\subsection{Simple property of the trivial equilibrium}

By linearizing system (6) at the origin (trivial equilibrium), one obtains the Jacobian

$$
J_{0}=\left(\begin{array}{lll}
a & 0 & 0 \\
b & d & 0 \\
0 & 0 & e
\end{array}\right)
$$

The eigenvalues of matrix $J_{0}$ are

$$
\lambda_{01}=a, \lambda_{02}=d, \lambda_{03}=e .
$$

Remark 2 As a,d,e $\in R$, there is no imaginary eigenvalue in (17), and a Hopf bifurcation does not exist near the trivial equilibrium which is different to systems (2), (4) and (5). The dynamics near the origin is simpler than for systems (2), (4) and (5).

\subsection{Symmetry and similarity}

It is obvious that the new system is symmetric about $\mathrm{z}$-axis, which can be easily proven via the transformation $(x, y, z) \rightarrow(-x,-y, z)$. Equilibria $S_{1}, S_{2}$ are also symmetric with respect to the $z$-axis and the same goes for $S_{3}, S_{4}$.

The dynamics near the neighborhood of $S_{1}, S_{2}$ is similar to each other in system (6), this feature also applies to $S_{3}, S_{4}$, and is caused by the similarity of the Jacobians of $S_{1}$ and $S_{2}$ $\left(S_{3}\right.$ and $\left.S_{4}\right)$.

To prove this, let $J_{i}$ denote the Jacobian of $S_{i}, i=1, \cdots, 4$, namely

$$
\begin{aligned}
J_{1} & =\left(\begin{array}{ccc}
a & c z_{e}^{1} & c_{1} y_{e} \\
b-z_{e}^{1} & d & \frac{c}{a} y_{e} z_{e}^{1} \\
f y_{e} & -f \frac{c}{a} y_{e} z_{e}^{1} & e
\end{array}\right), J_{2}=\left(\begin{array}{ccc}
a & c z_{e}^{1} & -c y_{e} \\
b-z_{e}^{1} & d & -\frac{c}{a} y_{e} z_{e}^{1} \\
-f y_{e} & f \frac{c}{a} y_{e} z_{e}^{1} & e
\end{array}\right), \\
J_{3} & =\left(\begin{array}{ccc}
a & c z_{e}^{2} & c y_{e} \\
b-z_{e}^{2} & d & \frac{c}{a} y_{e} z_{e}^{2} \\
f y_{e} & -f \frac{c}{a} y_{e} z_{e}^{2} & e
\end{array}\right), J_{4}=\left(\begin{array}{ccc}
a & c z_{e}^{2} & -c y_{e} \\
b-z_{e}^{2} & d & -\frac{c}{a} y_{e} z_{e}^{2} \\
-f y_{e} & f \frac{c}{a} y_{e} z_{e}^{2} & e
\end{array}\right) .
\end{aligned}
$$

There is a transformation matrix $T$, so that

$$
T^{-1} J_{1} T=J_{2}, T^{-1} J_{3} T=J_{4},
$$

where $T=\operatorname{diag}\left(\begin{array}{lll}-1 & -1 & 1\end{array}\right)$, and $T$ is an orthogonal ma- 


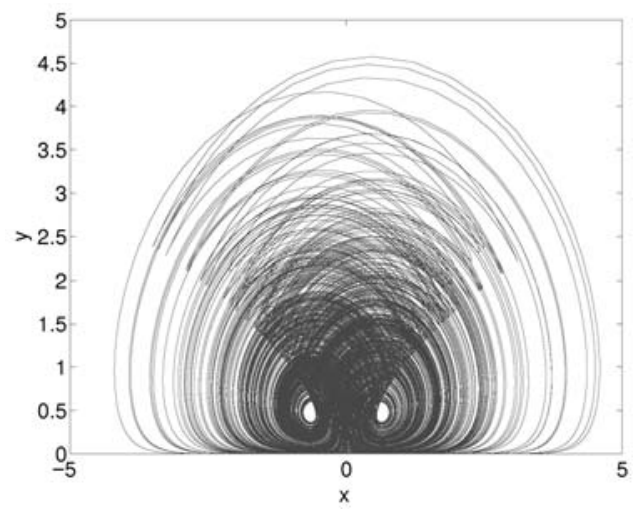

(a) Projection on the $x-y$ plane with an initial state $(1,1,1)$

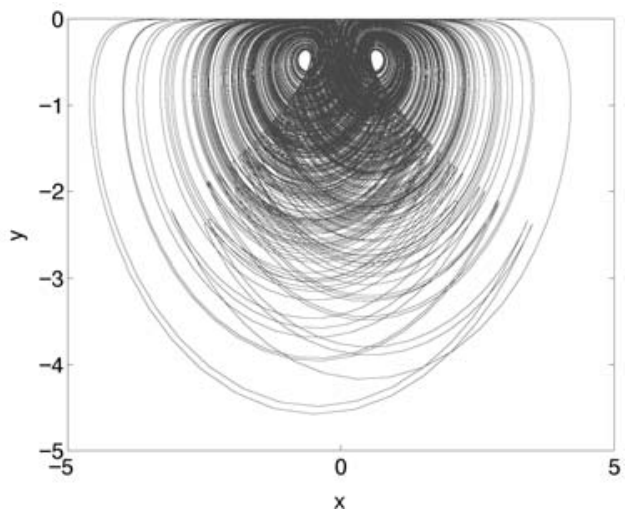

(b) Projection on the $x-y$ plane with an initial state $(-1,-1,1)$

FIG. 4: Double-wing chaotic attractor of (6), with $a=0.2, b=0, c=1, d=-0.4, e=-1.0$ and $f=-1$.

trix, since

$$
T^{-1}=T^{T}=T
$$

Let $V_{i}=\left[v_{i 1}, v_{i 2}, v_{i 3}\right]$ be the matrix consisted of eigenvectors of $S_{i}, i=1, \cdots, 4$, the following equations can be obtained:

$$
T^{-1} V_{1} T=V_{2}, \quad T^{-1} V_{3} T=V_{4}
$$

Remark $3 S_{1,2}$ and $S_{3,4}$ are two distinct equilibrium-pairs, and every equilibrium-pair has the same local stable, unstable and center manifolds.

\section{THE FOUR-WING CHAOTIC ATTRACTOR}

\subsection{Bifurcation analysis with respect to parameter $b$}

When $a=0.2, b=-0.01, c=1, d=-0.4, e=-1.0$ and $f=-1$, there are five equilibria in system (6), namely

$$
\begin{aligned}
& S_{0}=(0,0,0), S_{1}=(-0.6214,0.4472,0.2779) \\
& S_{2}=(0.6214,-0.4472,0.2779) \\
& S_{3}=(0.6437,0.4472,-0.2879) \\
& S_{4}=(0.6214,-0.4472,-0.2879)
\end{aligned}
$$

As $\nabla V=\frac{\partial \dot{x}}{x}+\frac{\partial \dot{y}}{y}+\frac{\partial \dot{z}}{z}=a+d+e=-1.2<0$, the system is dissipative. In order to investigate the stability of all the equilibria, we consider the Jacobian matrix with respect to each equilibrium and calculate their eigenvalues. The results are shown in Table I. Based on the eigenvalues, we know that $S_{0}, S_{1}, S_{2}, S_{3}$ and $S_{4}$ are all saddle-focus nodes implying that all the five equilibria are unstable.

In order to determine whether the system is chaotic or not, Lyapunov exponents should be calculated. In this paper, we use the efficient QR based method [30] to compute the Lyapunov exponents or Lyapunov exponent spectrum. With these parameter values, the corresponding Lyapunov exponents are $\lambda_{1}=0.064, \lambda_{2}=0$ and $\lambda_{3}=-1.262$ for system (6) and the system exhibits the four-wing chaotic dynamics which is shown in Fig. 1. The projections of the phase portrait on the $x-y, x-z$ and $y-z$ planes are shown in Fig. $1(\mathrm{a})-1(\mathrm{c})$, respectively. The 3 -D chaotic attractor is shown in Fig. 1(d).

The system equilibria $S_{i}, i=1, \cdots, 4$, which are denoted as red '*', are located at the centers of the four wings of the attractor, and the origin is indicated by the red symbol 'o', which is in the center of the whole chaotic attractor shown in Fig.1. It can be seen that there exist many orbits not only around $S_{1,2}$, but also around $S_{3,4}$, and even around $S_{1,3}$ and $S_{2,4}$, which play an important role in forming the real fourwing attractor, since they effectively connect the four subattractors, which surround the four equilibria.

Fig. 3 shows the maximum Lyapunov exponent spectrum, which corresponds directly to the bifurcation diagram shown in Fig. 2. Seen from Theorem 1, the parameter $b$ is a very important factor to create a four-wing attractor. Fig. 2 shows the bifurcation diagram of the state variable $x$, in which the orbit starts from $(1,1,1)$.

As can be seen from Fig. 2 and Fig. 3, the chaotic attractors are symmetrical about parameter $b$ and the middle point is $b=0$, which implies that parameter $b$ has little effect on the chaotic dynamics, except for causing system (6) to exhibit a four-wing attractor.

There are two kinds of orbital dynamical attractors in systems (6), a local one and a global one. The local attractor relies on the initial region of the orbit, which includes a sink, some simple periodic orbits and a single-wing chaotic attractor. The global attractor, which includes some complicated orbits around all equilibria, a double-wing chaotic attractor and a four-wing chaotic attractor, does not rely on the initial region of the orbit. An obvious illustration is the phase figures when $b=0$. When $a=0.2, b=0, c=1, d=-0.4, e=$ -1.0 and $f=-1$, the phase diagrams of (6) are shown in Fig. 4. In Fig. 4(a), the initial state is $(1,1,1)$ which is different from Fig. 4(b) with an initial state $(-1,-1,1)$. As can be seen from Fig. 4, they can not generate four-wing chaotic attractors when $b=0$, satisfying Theorem 1, but create two coexisting double-wing chaotic attractors. 
TABLE I: Eigenvalues of Jacobian matrixes for all equilibria.

\begin{tabular}{lllll}
\hline$S_{0}$ & \multicolumn{1}{c}{$S_{1}$} & \multicolumn{1}{c}{$S_{2}$} & \multicolumn{1}{c}{$S_{3}$} & \multicolumn{1}{c}{$S_{4}$} \\
\hline$\lambda_{1}=-0.4$ & $\lambda_{1}=-1.36$ & $\lambda_{1}=-1.36$ & $\lambda_{1}=-1.38$ & $\lambda_{1}=-1.38$ \\
$\lambda_{2}=0.2$ & $\lambda_{2,3}=0.08 \pm 0.47 i$ & $\lambda_{2,3}=0.08 \pm 0.47 i$ & $\lambda_{2,3}=0.09 \pm 0.48 i$ & $\lambda_{2,3}=0.09 \pm 0.48 i$ \\
$\lambda_{3}=-1$ & & & & \\
\hline
\end{tabular}

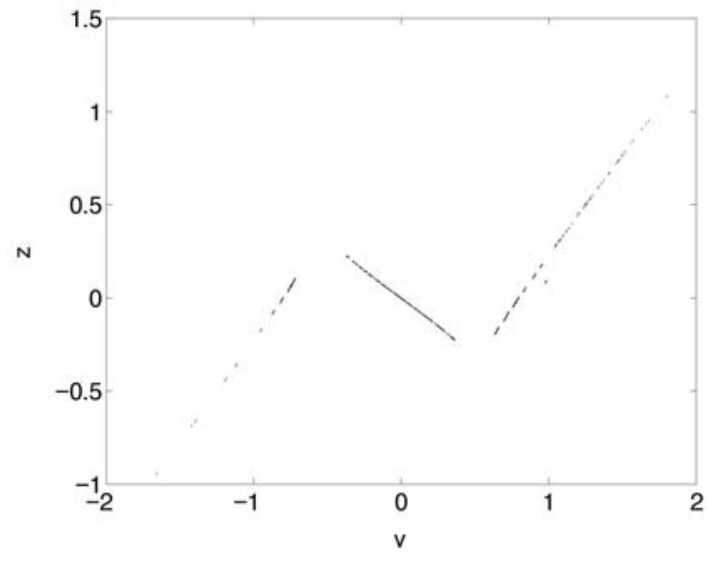

(a) Poincaré map on the crossing section $x=-0.62$

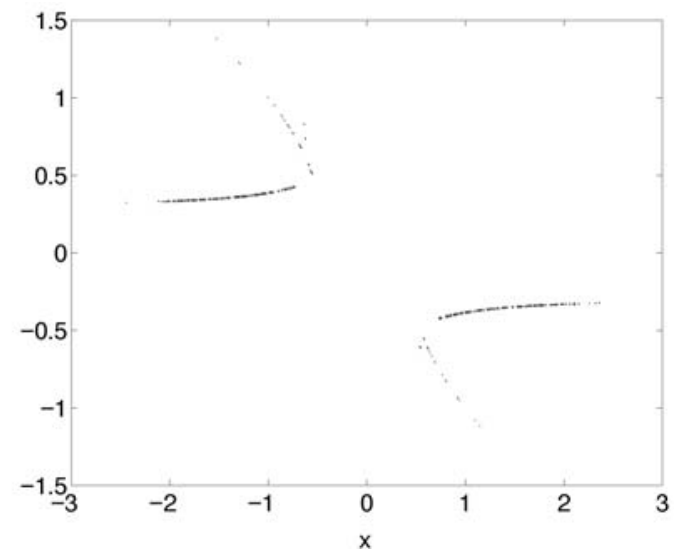

(c) Poincaré map on the crossing section $z=0.28$

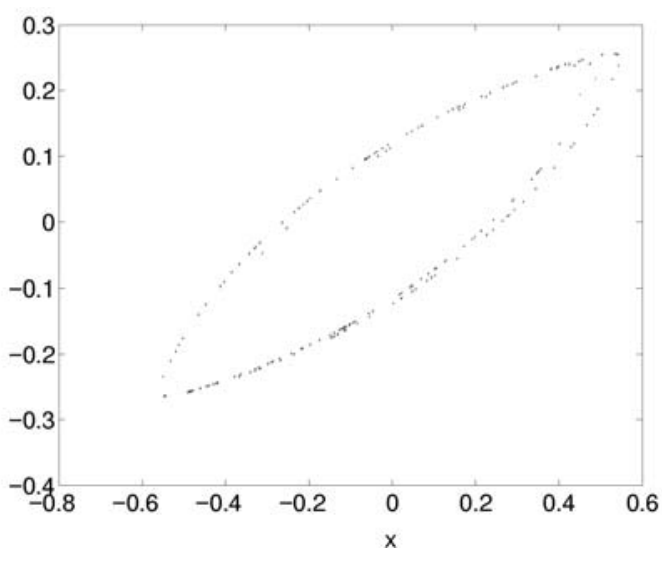

(b) Poincaré map on the crossing section $y=-0.45$

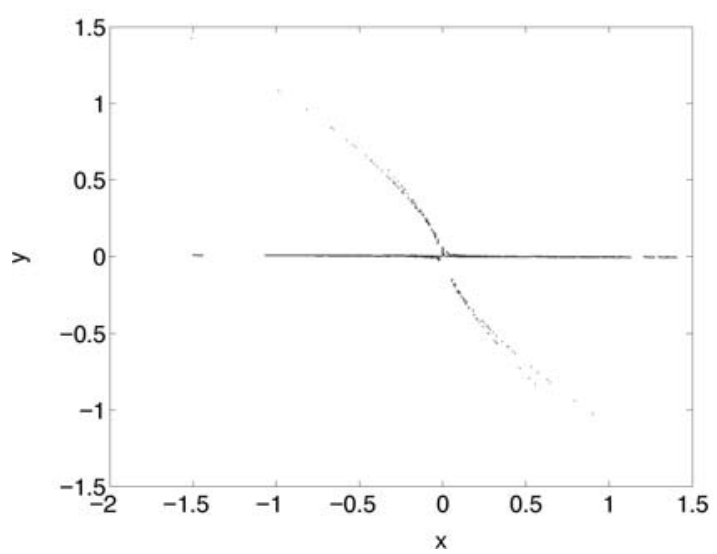

(d) Poincaré map on the crossing section $z=0$

FIG. 5: Four-wing chaotic attractor Poincaré mappings: with $a=0.2, b=-0.01, c=1, d=-0.4, e=-1.0$ and $f=-1$.

\subsection{Poincare map of the four-wing chaotic attractor}

As an important analysis technique, the Poincaré map can reflect bifurcation and folding properties of chaos. When $a=$ $0.2, b=-0.01, c=1, d=-0.4, e=-1.0$ and $f=-1$, one may take $x=-0.62, y=-0.45, z=0.28$ and $z=0$ as crossing planes, respectively, where $x=-0.62, y=-0.45, z=$ 0.28 is near the elements of the equilibrium of system (6). Fig. 5 shows the Poincaré mapping on several sections, with several sheets of the attractors visualized. It is clear that some sheets are folded and and indicates that the system has extremely rich dynamics.

\section{CONCLUSION}

Several 3-D four-wing smooth quadratic autonomous chaotic systems were analyzed and it was found that these systems have similar features related to the creation of four- wing chaotic attractors. A 3-D continuous autonomous system with less terms was consequently introduced. Some basic properties of the new system were also analyzed by means of Lyapunov exponents, bifurcation diagrams and Poincare maps. Phase diagrams showed that the equilibria are related to the existence of several wings. The new system is very convenient for understanding the dynamical behavior of multi-wing chaotic systems.

\section{ACKNOWLEDGMENT}

This work was supported by the grants: National Research Foundation of South Africa (No. IFR2008111000017); Tshwane University Research foundation, South Africa; the Natural Science Foundation of China (No. 10772135, 60774088); the Scientific Foundation of Tianjin City, China (No. 07JCYBJC05800) and the Scientific and Technological Research Foundation of Ministry of Education, China (No. 
207005).

[1] E. N. Lorenz, J. Atmos. Sci. 20, 130 (1963).

[2] A. S. de Paula and M. A. Savi, Brazilian Journal of Physics 38, 536 (2008).

[3] D. M. Maranhao and C. P. C. Prado, Brazilian Journal of Physics 35, 162 (2005).

[4] L. O. Chua, M. Komuro and T. Matsumoto, IEEE Trans Circuits Syst-I 33, 1072 (1986).

[5] L. O. Chua and T. Roska, IEEE Trans Circuits Syst-I 40, 147 (1993).

[6] J. A. K. Suykens and L. O. Chua, Int. J. Bif. Chaos 7, 1873 (1997).

[7] J. A. K. Suykens and J. Vandewalle, IEEE Trans. Circuits Syst-I 40, 861 (1993).

[8] K. S. Tang, G. Q. Zhong, G. Chen and K. F. Man, IEEE Trans Circuits Syst-I 48, 1369 (2001).

[9] M. E. Yalcin, S. Ozoguz, J. A. K. Suykens and J. Vandewalle, Electron. Lett. 37, 147 (2001).

[10] M. E. Yalcin, J. A. K. Suykens, J. Vandewalle and S. Ozoguz, Int. J. Bif. Chaos 12, 23 (2002).

[11] J .Lü, X. Yu and G. Chen, IEEE Trans Circuits Syst-I 50, 198 (2003).

[12] J. Lü, F. Han, X. Yu and G. Chen, Automatica 40, 1677 (2004).

[13] J. Lü, G. Chen and X. Yu, IEEE Trans. Circuits Syst-I 51, 2476 (2004).

[14] F. Han, X. Yu, J. Lü, G. Chen and Y. Feng, Dynam. Continuous Discrete Impulse Syst Ser B: Appl. Algorith. 12, 95 (2005).

[15] G. Y. Qi, G. R. Chen, M. A. van Wyk and B. J. van Wyk et al,
Chaos, Solitions Frac. 38, 705 (2008).

[16] G. Y. Qi, G. Chen, S. W. Li and Y. H. Zhang, Int. J. Bif. Chaos 16, 859 (2006)

[17] A. Vanečěk and S. Čelikovský, Control systems from linear analysis to synthesis of chaos, (Prentice-Hall, London, 1996).

[18] G. Chen and T. Ueta, Int. J. Bif. Chaos 9, 1465 (1999).

[19] G. Chen and J. Lü, Dynamical analysis, control and synchronization of the generalized Lorenz systems family, (Beijing, Science Press, 2003).

[20] S. Čelikovský and G. Chen, Chaos, Solitons Fract. 26, 1271 (2005).

[21] G. Y. Qi, S. Z. Du, G. Chen, Z. Q. Chen and Z. Z. Yuan, Chaos, Solitons Fract. 23, 1671 (2005).

[22] G. Y. Qi, B. J. van Wyk and M. A. van Wyk, Chaos, Solitons Fract. doi:10.1016/j.chaos.2007.09.095 (2007).

[23] A. S. Elwakil, S. Özoğz and M. P. Kennedy, Int. J. Bif. Chaos 13, 3093 (2003).

[24] W. B. Liu and G. Chen, Int. J. Bif. Chaos 13, 261 (2003).

[25] W. B. Liu and G. Chen, Int. J. Bif. Chaos 14, 971 (2004).

[26] W. B. Liu and G. Chen, Int. J. Bif. Chaos 14, 1395 (2004).

[27] J. Lü, Int. J. Bif. Chaos 14, 1507 (2004).

[28] T. S. Zhou and G. Chen, Int. J. Bif. Chaos 16, 2459 (2006).

[29] Z. Chen, Y. Yang and Z. Yuan, Chaos, Solitons Fract. 38:1187 (2008).

[30] H. F. von Bremen, F. E. Udwadia and W. Proskurowski, Physica D 101, 1 (1997). 